\title{
A Sensitive and Simple Method to Assess NK Cell Activity by RT-qPCR for Granzyme B Using Spleen and Blood
}

\author{
Fusako Mitsunaga ${ }^{1,2^{*}}$, Shin Nakamura ${ }^{1,2}$ \\ ${ }^{1}$ Intelligence and Technology Lab Inc., Kaizu, Japan \\ ${ }^{2}$ Biomedical Institute, NPO Primate Agora, Kaizu, Japan \\ Email: ${ }^{\star}$ mitsunaga@itechlab.co.jp
}

How to cite this paper: Mitsunaga, F. and Nakamura, S. (2021) A Sensitive and Simple Method to Assess NK Cell Activity by RT-qPCR for Granzyme B Using Spleen and Blood. Journal of Biosciences and Medicines, 9, 27-38.

https://doi.org/10.4236/jbm.2021.93004

Received: February 14, 2021

Accepted: March 15, 2021

Published: March 18, 2021

Copyright $\odot 2021$ by author(s) and Scientific Research Publishing Inc. This work is licensed under the Creative Commons Attribution International License (CC BY 4.0).

http://creativecommons.org/licenses/by/4.0/

\begin{abstract}
A new assay method for natural killer (NK) cell activity was established using quantitative RT-PCR (RT-qPCR) to determine the gene expression of granzyme B (GzmB) and perforin 1 (Prf1). The RT-qPCR method was compared to a conventional cytotoxic assay. Upregulated expression of GzmB and Prf1 mRNA and enhanced cytotoxic activity were observed in splenocytes from lipopolysaccharide (LPS)-treated rats. A high correlation, $\mathrm{R}^{2}=0.71$, was observed between the gene expression of GzmB and the cytotoxic activity of splenocytes from these rats, indicating that GzmB RT-qPCR is a reliable alternative method to assess NK cell activity/activation. Remarkably, 12.6- to 59.7-fold upregulation of GzmB mRNA expression was observed in leukocytes, the spleen, and splenocytes from LPS-injected rats. Its upregulation appeared to be dose-dependent on the LPS concentration in the range of 0.01 $0.1 \mathrm{mg} / \mathrm{kg}$. Whereas, only 1.3 - to 1.9 -fold increase of cytotoxic activity was detected in splenocytes from the rats treated with LPS in the same range. From these, it is evident that, to assess NK cell activity/activation, the GzmB RT-qPCR method is highly sensitive compared with the conventional cytological assay. Furthermore, this GzmB RT-qPCR method is advantageous, as it does not require freshly prepared splenocytes and cell culture procedures. The convenience of GzmB RT-qPCR enables the use of whole blood, leukocytes, the spleen, and/or their frozen samples to evaluate NK cell activity/activation.
\end{abstract}

\section{Keywords}

NK Activation, Functional Foods, Immunostimulatory Effect, mRNA, Frozen Samples 


\section{Introduction}

NK cells participate in eliminating tumor cells and virus-infected cells through their cytotoxic activity. Activated NK cells display enhanced cytotoxic activity toward target cells; hence, activation of NK cells is critical for effective cancer immunotherapy and protection against viral infections [1]. Previously, a chromium release assay was typically used to measure the NK cell activity [2]. In this assay, target cells are labeled with radioactive $51 \mathrm{Cr}$, and the cytotoxicity of $\mathrm{NK}$ cells as effector cells is assayed by detecting the release of radioisotopes from target cells. Since the use of radioactive isotopes is biohazardous, alternative methods are required. Labeling of target cells with nonradioactive europium is one such improvement [3]. Lately, cytotoxic lactate dehydrogenase and WST-1/ WST-8 cell proliferation assays have been employed to measure NK activity [4] [5]. These assays do not require the labeling of target cells, and commercially available kits can be used. However, they still require time-consuming cytological procedures to prepare fresh splenocytes as effector cells and their co-culture with target cells. Thus, another simple and easy method for measuring NK cell activity is required.

Cytotoxic activity of NK cells is mediated by effector molecules such as granzymes (a member of the lysosomal serine protease family), especially granzyme B (GzmB), and perforin (Prf1) [6]. Prf1 facilitates the entry of GzmB into target cells, resulting in the induction of apoptosis. Thus, GzmB and Prf1 appear to be possible markers for assessing the cytotoxic activity of NK cells. In resting NK cells, low mRNA expression levels of GzmB and Prf1 were observed [7]. It has been reported that NK cell activation is accompanied by an increase in the mRNA levels of these genes [8] [9]. These suggest that the use of RT-qPCR to determine the mRNA expression of GzmB and/or Prf1 is an alternative method to assess NK cell activity/activation instead of the conventional cytological method.

In the present study, we performed RT-qPCR to determine the mRNA expression of GzmB and/or Prf1 to assess NK cell activity/activation of splenocytes, the spleen, leukocytes, and whole blood from lipopolysaccharide (LPS)treated rats and mice. The RT-qPCR method was highly sensitive, useful, and applicable for evaluating the efficiency of NK cell activity/activation of foods and drugs.

\section{Materials and Methods}

\subsection{Animals and Treatment}

Sprague-Dawley (SD) and Wistar rats and ICR mice were purchased from Japan SLC Inc. (Hamamatsu, Japan). LPS from E. coli O55 (phenolic extraction) was purchased from FUJIFILM Wako Pure Chemical Corporation (Osaka, Japan). Animals were housed in groups of 2 - 6 individuals in stainless steel cages under control conditions of a $12 / 12 \mathrm{~h}$ light/dark cycle at $20^{\circ} \mathrm{C}-28^{\circ} \mathrm{C}$ with a relative humidity of $30 \%-80 \%$. They had free access to tap water and a standard rodent 
diet (MF, Oriental Yeast, Tokyo, Japan). All experimental procedures were approved by the Institutional Animal Ethics Committee in accordance with the institutional guidelines based on the Guide for the Care and Use of Laboratory Animals by the National Research Council (USA) [10].

After more than 1 month of acclimatization, the rats and mice were injected with LPS. LPS was diluted with saline and intravenously administered via the tail vein at $0.01,0.03$, and $0.1 \mathrm{mg} / \mathrm{kg}$ body weight (BW). Control individuals were injected with saline. Wistar rats $(350-450 \mathrm{~g})$ and SD rats $(450-550 \mathrm{~g})$ were allocated to examine the cytotoxic activity of splenocytes and gene expression of peripheral leukocytes, splenocytes, and spleen tissue. ICR mice (30 - $40 \mathrm{~g})$ were allocated to determine gene expression in whole blood and spleen tissues.

\subsection{Tissue and Blood Sample Collection}

In both rat and mouse experiments, blood samples were collected under anesthesia $2 \mathrm{~h}$ after LPS administration, followed by euthanization and spleen isolation.

In the rat experiment, blood samples were collected in heparinized syringes, and leukocytes were isolated using erythrocyte lysis buffer, as described by Hoffman et al. [11]. The spleen was removed, and splenocytes were harvested by passing the cells through a nylon mesh cell strainer (Falcon-Corning, Corning, NY, USA). Splenocytes were further purified using Hoffman lysis buffer. The obtained splenocytes were used for the NK cell activity test and RT-qPCR. Splenocytes for RT-qPCR were washed with phosphate-buffered saline, lysed, and homogenized in RNAiso Plus (Takara Bio Inc., Kusatsu, Japan). A portion of the spleen was sliced and treated with RNAlater (Sigma-Aldrich, St. Louis, MO, USA) immediately after tissue removal. Treated tissue samples were stored at $-70^{\circ} \mathrm{C}$ until RNA extraction.

In the mouse experiment, blood samples were collected in heparinized syringes, treated with Buffer DL from the NucleoSpin RNA Blood Kit (Macherey-Nagel GmbH \& Co. KG, Düren, Germany), and kept frozen at $-70^{\circ} \mathrm{C}$ until RNA extraction. The removed spleen was treated with RNAlater and processed as described above.

\subsection{Cytotoxic Activity Assay of NK Cells from Splenocytes Using the WST-1 Assay}

YAC-1 cells (mouse lymphoma origin, KAC Co., Ltd., Kyoto, Japan) were used as target cells to test the cytotoxic activity of NK cells. Harvested rat splenocytes were examined for their cytotoxic ability as effector cells (E) against target cells (T). Splenocytes and YAC-1 cells were co-cultured at a 1:1 ratio of E:T $(1 \times 106$ cells $/ \mathrm{mL}$ ) and were also cultured independently in 96-well plates for $4 \mathrm{~h}$ in a $5 \%$ $\mathrm{CO}_{2}$ atmosphere at $37^{\circ} \mathrm{C}$. Premix WST-1 (Takara Bio Inc.) was added to the wells and incubated for $60 \mathrm{~min}$. The absorbance was measured at $450 \mathrm{~nm}$ using a plate reader (iMark Microplate Reader, Bio-Rad, Hercules, CA, USA). The cyto- 
toxic activity of NK cells was calculated using the standard formula, 1 - (ET $\mathrm{E} / \mathrm{T}) \times 100(\%)[5]$.

\subsection{Total RNA Isolation}

Total RNA was extracted from peripheral leukocytes, splenocytes, and spleen tissues as described previously using the acid guanidine thiocyanate-phenolchloroform method and silica membrane column-based purification [12]. Briefly, cells or tissues were homogenized in RNAiso Plus (Takara Bio Inc.), and the total RNA was isolated. The RNA was treated with DNase (Qiagen, Valencia, CA, USA) in the aqueous phase and further purified using the RNeasy MinElute Cleanup Kit (Qiagen) according to the manufacturer's instructions.

Total RNA (0.2 - $0.4 \mathrm{~mL})$ of mouse whole blood was isolated using NucleoSpin RNA Blood (Macherey-Nagel GmbH \& Co.) according to the manufacturer's instructions with on-column DNA digestion.

The quantity and purity of RNA were evaluated at 230, 260, 280, and $320 \mathrm{~nm}$ using an Ultrospec 2000 spectrometer (GE Healthcare Bio-Sciences AB, Uppsala, Sweden).

\subsection{RT-qPCR of GzmB and Prf1}

The mRNA levels of GzmB and Prf1 in tissue and cell samples were determined by RT-qPCR, as described previously [12]. cDNA was synthesized from the purified RNA using PrimeScript Reverse Transcriptase (Takara Bio Inc.) with RNase Inhibitor (Takara Bio Inc.), dNTP mixture (Promega Corp., Madison, WI, USA.), and Oligo dT primers (Invitrogen, Waltham, MA, USA).

Real-time PCR was performed using the Mx3000P QPCR System (Agilent Technologies Inc., Santa Clara, CA, USA) with a SYBR Premix Ex Taq II (Tli RNaseH Plus) Kit (Takara Bio Inc.). Specific primers for rat and mouse GzmB, Prfl, and the reference gene low-density lipoprotein receptor-related protein 10 (Lrp10) were constructed using Primer3 and Primer-BLAST [13]. A standard curve was generated by serial dilution of a known amount of GAPDH amplicon to calculate the cDNA copy number of the genes [12]. The PCR conditions were as follows: $95^{\circ} \mathrm{C}$ for $15 \mathrm{~s}$, followed by 35 cycles of $95^{\circ} \mathrm{C}$ for $10 \mathrm{~s}$ and $63^{\circ} \mathrm{C}$ for $30 \mathrm{~s}$, with a dissociation curve. The quantity of GzmB or Prf1 mRNA was expressed as the ratio against that of a suitable reference gene, Lrp10 [14].

\section{Results}

\subsection{Cytotoxic Activity of NK Cells of Splenocytes from Untreated and LPS-Injected Rats}

Table 1 shows the cytotoxic activity of NK cells in splenocytes from SD and Wistar rats. Their cytotoxic activity was enhanced by $1.3-$ to 1.9 -fold in splenocytes from LPS-treated animals and increased in a dose-dependent manner with the LPS concentration from $0.01-0.1 \mathrm{mg} / \mathrm{kg}$. Figure 1 represents an average result, in which the NK cell cytotoxicity of splenocytes from both rats increased 
Table 1. Cytotoxic activity of NK cell in splenocyte from LPS-injected SD and wister rats.

\begin{tabular}{ccc}
\hline \multirow{2}{*}{ LPS (mg/kg B.W.) } & \multicolumn{2}{c}{ NK cell activity $1-(\mathrm{ET} /(\mathrm{E}+\mathrm{T}))$} \\
\cline { 2 - 3 } & $\mathrm{SD}$ & Wister \\
\hline 0 (Saline Control) & 24.0 & 16.3 \\
0.01 & 25.4 & 25.1 \\
0.03 & 32.5 & 35.6 \\
0.10 & 40.0 & 35.6 \\
\hline
\end{tabular}

Splenocytes were isolated from LSP-treated SD and Wister rats. The cytotoxic activity of NK cell (NK cell activity) was assayed under the conditions as mentioned in Materials and methods.

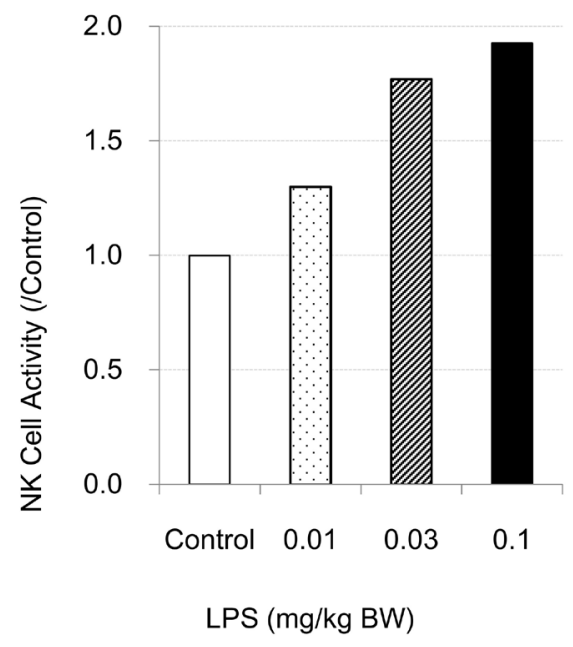

Figure 1. Mean cytotoxic activity of NK cell (NK cell activity) in splenocytes from LPS-treated SD and Wister rats and its dose-dependency with LPS injected.

dose-dependently with LPS concentration.

\subsection{Alternative Method to Assess NK Cell Cytotoxicity Using RT-qPCR}

An alternative assay method to measure the cytotoxic activity of NK cells was examined by means of RT-qPCR to determine gene expression, i.e. steady-state mRNA level, of both GzmB and Prf1. The expression levels of GzmB and Prf1 mRNA were dose-dependently upregulated in splenocytes from LPS-treated rats and mice (Table $2 \&$ Table 3, Figures 3-5). Figure 2 shows a significant correlation $\left(R^{2}=0.71\right)$ between the gene expression of GzmB and the cytotoxic activity of splenocytes from rats. This indicates that RT-qPCR for quantitative analysis of mRNA expression of GzmB is a reliable method for cytotoxic assays of NK cells.

\subsection{LPS Upregulated the Gene Expression of GzmB and Prf1}

As shown in Table 2 and Table 3, LPS-mediated upregulation of both GzmB and Prf1 mRNA was observed in leukocytes from peripheral blood, splenocytes, 
Table 2. Relative gene expression (mRNA levels) of GzmB in samples from LPS-treated rats.

\begin{tabular}{cccc}
\hline \multirow{2}{*}{ Samples } & LPS (mg/kg B.W.) & \multicolumn{2}{c}{ GzmB/Lrp10 } \\
\cline { 3 - 4 } Leukocytes & 0 (saline control) & 1.9 & Wister \\
& 0.01 & 7.3 & 1.5 \\
& 0.03 & 38.5 & 75.8 \\
& 0.10 & 12.1 & 8.7 \\
Splenocytes & 0 (saline control) & 2.7 & 28.9 \\
& 0.01 & 150.7 & 9.0 \\
& 0.03 & 179.4 & 40.7 \\
Spleen & 0.10 & 74.2 & 136.5 \\
& 0 (saline control) & 0.4 & 215.9 \\
& 0.01 & 21.6 & 1.2 \\
& 0.03 & 41.2 & 15.8 \\
& 0.10 & 26.6 & 28.4 \\
& & & 39.9 \\
\hline
\end{tabular}

Blood sample and spleen were obtained 2 hours later of intravenous injection of LPS. White blood cells (leukocytes) were separated from whole blood and splenocytes were isolated from spleen by the procedures mentioned in materials and methods. RNA was extracted from these samples and RT-qPCR to quantify mRNA levels of both GzmB and Lrp10 genes were performed. Data are expressed as ratio of copy number of GzmB against that of a reference gene (Lrp10).

Table 3. Relative gene expression (mRNA levels) of Prf1 in samples from LPS-treated rats.

\begin{tabular}{cccc}
\hline \multirow{2}{*}{ Samples } & LPS (mg/kg B.W.) & \multicolumn{2}{c}{ Prf1/Lrp10 } \\
\cline { 3 - 4 } Leukocytes & 0 (saline control) & 4.4 & Wister \\
\hline & 0.01 & 4.1 & 6.9 \\
& 0.03 & 21.1 & 21.9 \\
& 0.10 & 4.9 & 3.8 \\
Splenocytes & 0 (saline control) & 5.1 & 8.9 \\
& 0.01 & 21.9 & 7.6 \\
& 0.03 & 23.1 & 5.9 \\
& 0.10 & 11.9 & 8.9 \\
Spleen & 0 (saline control) & 2.0 & 14.4 \\
& 0.01 & 4.4 & 2.5 \\
& 0.03 & 6.3 & 4.3 \\
& 0.10 & 4.1 & 4.4 \\
& & & 7.4 \\
\hline
\end{tabular}

Conditions for sample preparations, RNA extractions, and RT-qPCRs for Prf1 and Lrp10 genes were same as mentioned in Table 2. Data are expressed as ratio of copy number of Prf1 against that of a reference gene (Lrp10).

and spleens from LPS-treated rats. The upregulated expression of GzmB mRNA was also observed in the whole blood and spleens of LPS-injected mice (Figure 4(a) and Figure 4(b)). 


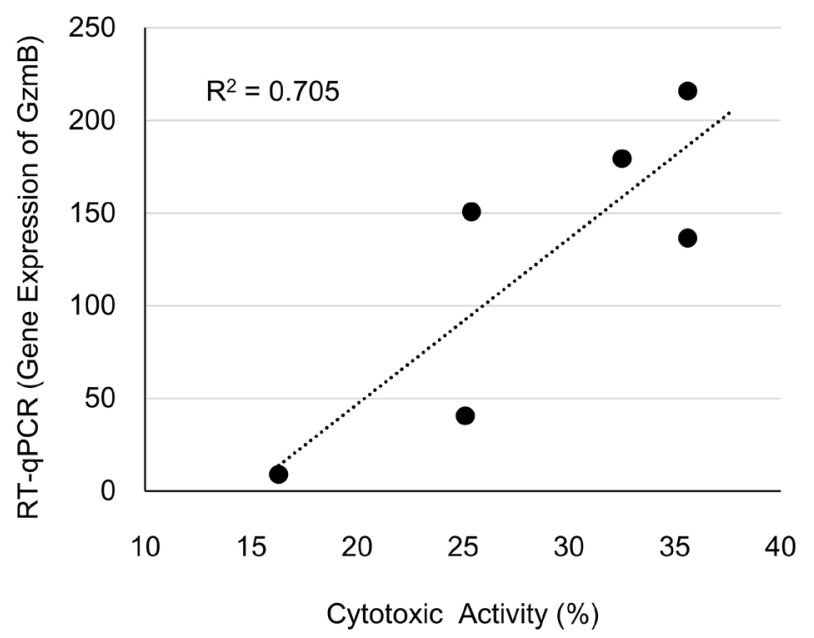

Figure 2. Correlation between cytotoxic activity and RT-qPCR (gene expression) of GzmB of NK cells in splenocytes from LPS-treated rats (LPS: 0 to $0.1 \mathrm{mg} / \mathrm{kg} \mathrm{BW}$ ). The cytotoxic activity was assayed and calculated as the conditions described in Materials and Methods. Gene expression of GzmB was expressed as ratio against reference gene (Lrp10).

Figure 3 shows the average relative expression of GzmB mRNA in both SD and Wister rats in comparison to the reference gene (Lrp10) in leukocytes (Figure 3(a)), splenocytes (Figure 3(b)), and spleen (Figure 3(c)). Remarkably, 12.6- to 59.7-fold upregulation of GzmB mRNA expression was observed in the three samples from rats administered LPS in the range of $0.01-0.1 \mathrm{mg} / \mathrm{kg} \mathrm{BW}$ (Figure 3).

Figure 4 shows the same remarkable upregulation, by more than 10 -fold, of GzmB mRNA in the whole blood (Figure 4(a)) and spleen (Figure 4(b)) of LPS-injected mice. These results indicate that using RT-qPCR to measure GzmB mRNA is a highly sensitive method to assess the activity of NK cells compared with conventional cytological assay using WST-1. These results also suggest that RT-qPCR of GzmB mRNA is an alternative method to be applied to not only splenocytes and spleen, but also leukocytes and whole blood.

The gene expression of Prf1 was also upregulated in LPS-treated rats and mice, although the upregulation of Prf1 mRNA was milder than that of GzmB. Figure 5 shows the relative gene expression of rat Prf1 in leukocytes (Figure 5(a)), splenocytes (Figure 5(b)), and spleens (Figure 5(c)), expressed as the average of values determined in both SD and Wistar rats. Figure 6 represents upregulated Prf1 mRNA expression in whole blood, but not in the spleen, from mice injected at a concentration of $0.1 \mathrm{mg} / \mathrm{kg} \mathrm{BW}$.

\section{Discussion}

Recently, there has been much interest in drugs, functional foods, and their materials with immunomodulatory effects, including the stimulation of NK cell activity [15] [16] [17] [18]. Therefore, a simple and non-radioactive method to assay NK cell activity is required to facilitate the development of both drugs and 


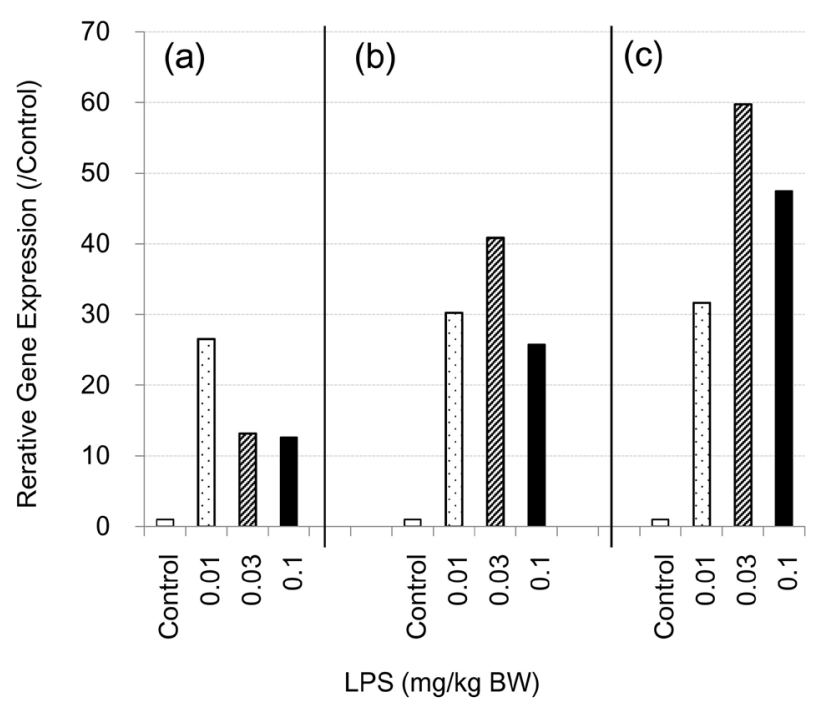

Figure 3. mRNA Levels of GzmB in (a) peripheral leukocytes, (b) isolated splenocytes, and (c) spleen from control and LSP-treated rats. The data from SD and Wister rats are averaged and expressed as ratio against saline control.

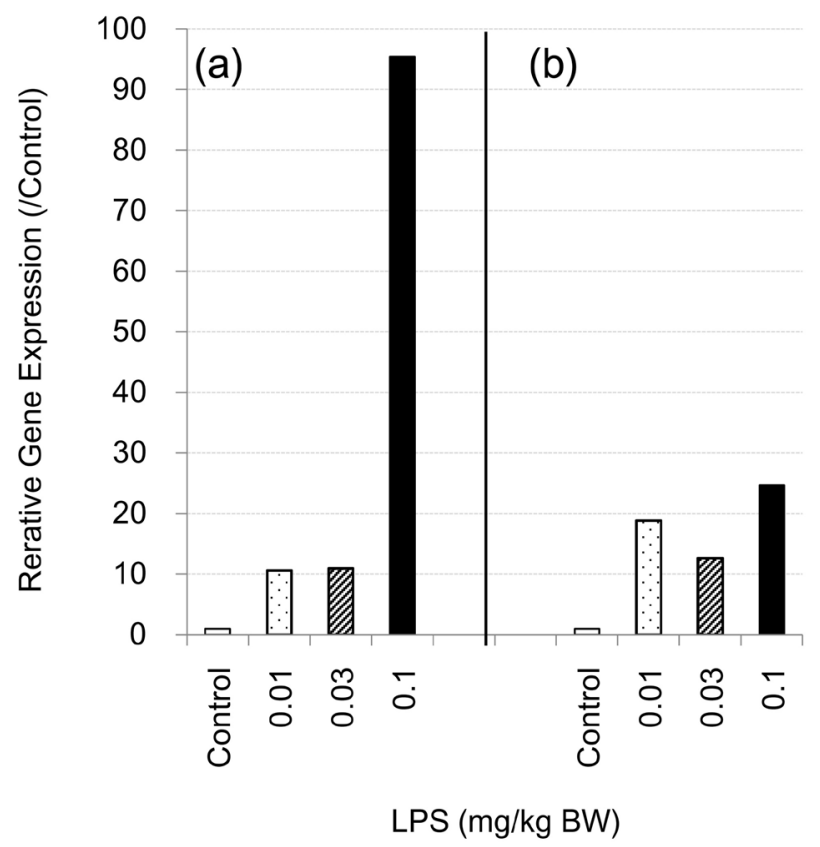

Figure 4. mRNA Levels of GzmB in (a) whole blood), (b) spleen from control and LPS-treated mice. The data are expressed as ratio against saline control.

foods with immunomodulatory action(s). Furthermore, a highly sensitive method is essential because functional foods and related materials can mildly affect NK cell activity/activation.

NK cells, which are effector lymphocytes of innate immunity, represent $10 \%$ $20 \%$ of peripheral blood mononuclear cells and play key roles in eliminating tumor cells and virus-infected cells [19] [20]. This self-defensive role of NK cells 


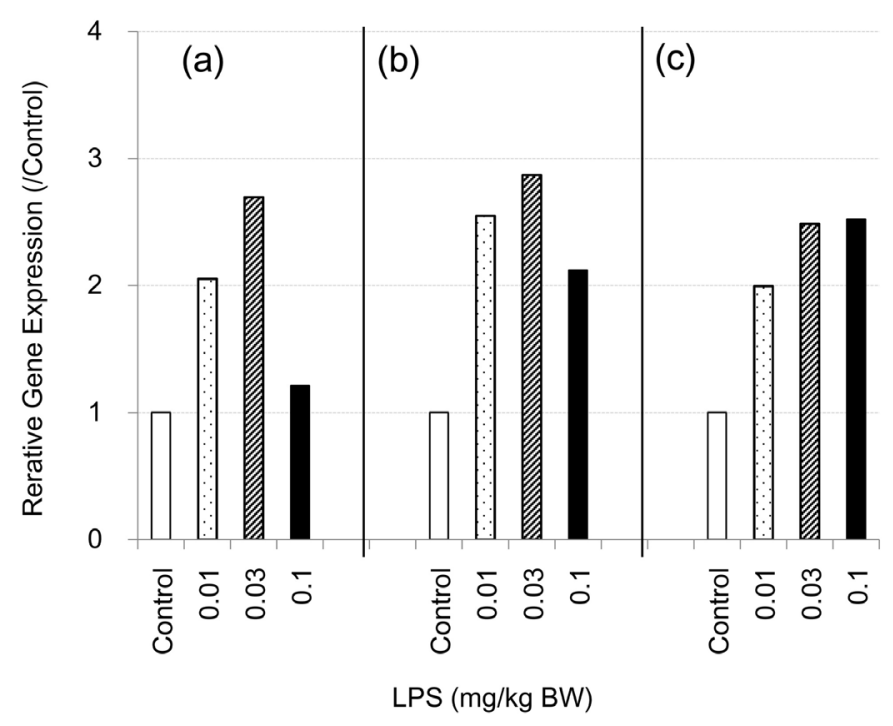

Figure 5. mRNA Levels of Prf1 in (a) peripheral leukocytes, (b) isolated splenocytes, and (c) spleen from control and LPS-treated rats. The data from SD and Wister rats are averaged and expressed as ratio against saline control.

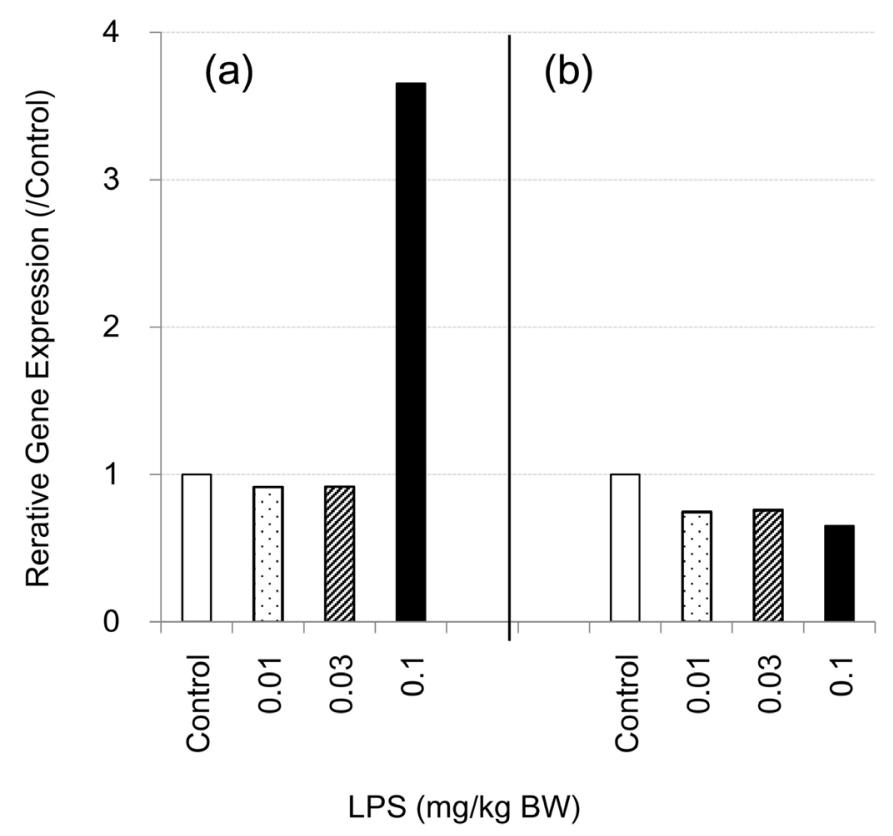

Figure 6. mRNA Levels of Prf1 in (a) whole blood), (b) spleen from control and LPS-treated mice. The data are expressed as ratio against saline control.

depends on their cytotoxic activity, which is mediated by the effector molecules GzmB and Prf1 [6].

The cytotoxicity of NK cells is regulated by transcriptional and/or translational events of GzmB and Prf1 and is known to be enhanced upon NK cell activation by several stimuli, including LPS [21].

In a previous report, resting NK cells expressed some basal levels of GzmB or 
Prf1 mRNA, although their protein levels were low [7]. The involvement of microRNAs is suggested to regulate the translation of these genes prior to posttranscriptional events [22] [23]. The enhanced cytotoxic activity of NK cells is mediated by their activation accompanied by an increase in the protein levels of these effectors. Transcription of these genes is upregulated upon activation of NK cells before translation. A kinetic study revealed that the upregulation of mRNA expression of GzmB and Prf1 preceded production of their proteins in IL-15-activated NK cells [22]. These results indicate that both upregulated mRNA expression and elevated protein synthesis of GzmB and Prf1 occur upon activation of NK cells. These results also lead us to consider new markers of GzmB and/or Prf1 mRNA and a molecular biological method, RT-qPCR, using these markers to assess NK cell activity/activation.

The expression levels of both GzmB and Prf1 mRNA were dose-dependently upregulated in splenocytes from LPS-treated rats and mice (Figure 3 and Figure 4). A significant correlation was observed between the gene expression of GzmB and the cytotoxic activity of splenocytes from LPS-treated rats (Figure 2). Thus, RT-qPCR of GzmB mRNA is a reliable method that can be used in place of conventional cytological assays for NK cell activity.

RT-qPCR of GzmB mRNA is a highly sensitive method for the assay of NK cell activity/activation. We observed a 41-fold upregulation of GzmB mRNA expression, whereas only a 1.9-fold increase in cytotoxic activity was detected in activated splenocytes from LPS-injected rats (Figure 1 and Figure 3(b)). A similar marked upregulation of GzmB mRNA was observed in leukocytes, spleen, and whole blood from LPS-injected rats and/or mice. This is evidence for the high sensitivity of GzmB RT-qPCR.

The other advantage of this RT-qPCR method is that it is unnecessary to prepare splenocytes from freshly isolated spleens. This makes it convenient to keep samples, spleens, leukocytes, and/or whole blood in a frozen state until use after treatment with RNAlater or related treatment. For these reasons, the GzmB RT-qPCR method can be applied to evaluate NK cell activity/activation in leukocytes and whole blood, in which NK cells comprise $10 \%-20 \%$ of peripheral blood mononuclear cells.

\section{Conclusions}

RT-qPCR to determine GzmB mRNA expression was established as an alternative method to assess NK cell activity/activation. This alternative GzmB RTqPCR method was highly sensitive and had high correlation with the conventional cytologic assay.

This GzmB RT-qPCR method made it unnecessary to prepare fresh splenocytes and co-culture with target cells. The alternative method enables the use of frozen samples, spleens, leukocytes, and/or whole blood. The GzmB RT-qPCR has enough sensitivity to detect mild effects on NK cell activity/activation by functional foods and their related materials. 


\section{Acknowledgements}

We thank Mr. Masaki Matsuura for his technical assistance with the assay of NK cytotoxic activity.

\section{Conflicts of Interest}

The authors declare no conflicts of interest regarding the publication of this paper.

\section{References}

[1] Rosenberg, J. and Huang, J. (2018) CD8+ T Cells and NK Cells: Parallel and Complementary Soldiers of Immunotherapy. Current Opinion in Chemical Engineering, 19, 9-20. https://doi.org/10.1016/j.coche.2017.11.006

[2] Brunner, K.T., Mauel, J., Cerottini, J.C. and Chapuis, B. (1968) Quantitative Assay of the Lytic Action of Immune Lymphoid Cells on 51-Cr-Labelled Allogeneic Target Cells in Vitro; Inhibition by Isoantibody and by Drugs. Immunology, 14, 181-196.

[3] von Zons, P., Crowley-Nowick, P., Friberg, D., Bell, M., Koldovsky, U. and Whiteside, T.L. (1997) Comparison of Europium and Chromium Release Assays: Cytotoxicity in Healthy Individuals and Patients with Cervical Carcinoma. Clinical and Diagnostic Laboratory Immunology, 4, 202-207.

https://doi.org/10.1128/CDLI.4.2.202-207.1997

[4] Choi, J.W., Lee, E.S., Kim, S.Y., Park, S.I., Oh, S., Kang, J.H., Ryu, H.A. and Lee, S. (2019) Cytotoxic Effects of ex Vivo-Expanded Natural Killer Cell-Enriched Lymphocytes (MYJ1633) against Liver Cancer. BMC Cancer, 19, 817. https://doi.org/10.1186/s12885-019-6034-1

[5] Ueda, H., Takeuchi, A. and Wako, T. (2013) Activation of Immune Responses in Mice by an Oral Administration of Bunching Onion (Allium fistulosum) Mucus. Bioscience, Biotechnology, and Biochemistry, 77, 1809-1813. https://doi.org/10.1271/bbb.130084

[6] Chowdhury, D. and Lieberman, J. (2008) Death by a Thousand Cuts: Granzyme Pathways of Programmed Cell Death. Annual Review of Immunology, 26, 389-420. https://doi.org/10.1146/annurev.immunol.26.021607.090404

[7] Fehniger, T.A., Cai, S.F., Cao, X., Bredemeyer, A.J., Presti, R.M., French, A.R. and Ley, T.J. (2007) Acquisition of Murine NK Cell Cytotoxicity Requires the Translation of a Pre-Existing Pool of Granzyme B and Perforin mRNAs. Immunity, 26, 798-811. https://doi.org/10.1016/j.immuni.2007.04.010

[8] Clement, M.V., Haddad, P., Soulie, A., Guillet, J. and Sasportes, M. (1990) Involvement of Granzyme B and Perforin Gene Expression in the Lytic Potential of Human Natural Killer Cells. Nouvelle revue francaise d hematologie, 32, 349-352.

[9] Salcedo, T.W., Azzoni, L., Wolf, S.F. and Perussia, B. (1993) Modulation of Perforin and Granzyme Messenger RNA Expression in Human Natural Killer Cells. Journal of Immunology (Baltimore, Md.: 1950), 151, 2511-2520.

[10] Institute for Laboratory Animal Research (1996) Guide for the Care and Use of Laboratory Animals National Research Council (US). National Academies Press (US), Washington DC. http://www.nap.edu/catalog/5140.html

[11] Hoffman, R.A., Kung, P.C., Hansen, W.P. and Goldstein, G. (1980) Simple and Rapid Measurement of Human T Lymphocytes and Their Subclasses in Peripheral Blood. Proceedings of the National Academy of Sciences of the United States of America, 77, 4914-4917. https://doi.org/10.1073/pnas.77.8.4914 
[12] Jeong, A.R., Nakamura, S. and Mitsunaga, F. (2008) Gene Expression Profile of Th1 and Th2 Cytokines and Their Receptors in Human and Nonhuman Primates. Journal of Medical Primatology, 37, 290-296. https://doi.org/10.1111/j.1600-0684.2008.00289.x

[13] Ye, J., Coulouris, G., Zaretskaya, I., Cutcutache, I., Rozen, S. and Madden, T.L. (2012) Primer-BLAST: A Tool to Design Target-Specific Primers for Polymerase Chain Reaction. BMC bioinformatics, 13, 134. https://doi.org/10.1186/1471-2105-13-134

[14] Gabrielsson, B.G., Olofsson, L.E., Sjögren, A., Jernås, M., Elander, A., Lönn, M., Rudemo, M. and Carlsson, L.M. (2005) Evaluation of Reference Genes for Studies of Gene Expression in Human Adipose Tissue. Obesity Research, 13, 649-652. https://doi.org/10.1038/oby.2005.72

[15] Hwang, Y.J., Kim, J., Park, D.S. and Hwang, K.A. (2012) Study on the Immunomodulation Effect of Isodon japonicus Extract via Splenocyte Function and NK Anti-Tumor Activity. International Journal of Molecular Sciences, 13, 4880-4888. https://doi.org/10.3390/ijms13044880

[16] Kim, Y., Kim, I., Park, C.H. and Kim, J.B. (2018) Korean Mistletoe Lectin Enhances Natural Killer Cell Cytotoxicity via Upregulation of Perforin Expression. Asian Pacific Journal of Allergy and Immunology, 36, 175-183.

https://doi.org/10.1016/j.jaci.2017.12.556

[17] Mohamad, N.E., Abu, N., Yeap, S.K., Lim, K.L., Romli, M.F., Sharifuddin, S.A., Long, K. and Alitheen, N.B. (2019) Apoptosis and Metastasis Inhibitory Potential of Pineapple Vinegar against Mouse Mammary Gland Cells in Vitro and in Vivo. Nutrition \& Metabolism, 16, 49. https://doi.org/10.1186/s12986-019-0380-5

[18] Kwon, H.Y., Choi, S.I., Han, X., Men, X., Jang, G.W., Choi, Y.E., Kim, S.H., Kang, J.C., Cho, J.H. and Lee, O.H. (2020) Enhancement of Immune Activities of Mixtures with Sasa Quelpaertensis Nakai and Ficus erecta var. sieboldii. Foods (Basel, Switzerland), 9, 868. https://doi.org/10.3390/foods9070868

[19] Herberman, R.B., Nunn, M.E. and Lavrin, D.H. (1975) Natural Cytotoxic Reactivity of Mouse Lymphoid Cells against Syngeneic Acid Allogeneic Tumors. I. Distribution of Reactivity and Specificity. International Journal of Cancer, 16, 216-229. https://doi.org/10.1002/ijc.2910160204

[20] Cooley, S., Parham, P. and Miller, J.S. (2018) Strategies to Activate NK Cells to Prevent Relapse and Induce Remission Following Hematopoietic Stem Cell Transplantation. Blood, 131, 1053-1062. https://doi.org/10.1182/blood-2017-08-752170

[21] Chen, C.P., Boyadjieva, N.I., Advis, J.P. and Sarkar, D.K. (2006) Ethanol Suppression of the Hypothalamic Proopiomelanocortin Level and the Splenic NK Cell Cytolytic Activity Is Associated with a Reduction in the Expression of Proinflammatory Cytokines But Not Anti-Inflammatory Cytokines in Neuroendocrine and Immune Cells. Alcoholism, Clinical and Experimental Research, 30, 1925-1932. https://doi.org/10.1111/j.1530-0277.2006.00237.x

[22] Kim, T.D., Lee, S.U., Yun, S., Sun, H.N., Lee, S.H., Kim, J.W., Kim, H.M., Park, S.K., Lee, C.W., Yoon, S.R., Greenberg, P.D. and Choi, I. (2011) Human microRNA-27a* Targets Prf1 and GzmB Expression to Regulate NK-Cell Cytotoxicity. Blood, 118, 5476-5486. https://doi.org/10.1182/blood-2011-04-347526

[23] Kim, N., Kim, M., Yun, S., Doh, J., Greenberg, P.D., Kim, T.D. and Choi, I. (2014) MicroRNA-150 Regulates the Cytotoxicity of Natural Killers by Targeting Perforin-1. The Journal of Allergy and Clinical Immunology, 134, 195-203. https://doi.org/10.1016/j.jaci.2014.02.018 